\title{
Density Based Traffic Signalling System using Image Processing
}

\author{
Adwait Sharma \\ Computer Department \\ St.John College of \\ Engineering \\ Mumbai, India
}

\author{
Aayush Soni \\ Computer Department \\ St.John College of \\ Engineering \\ Mumbai, India
}

\author{
Rahul Rajpurohit \\ Computer Department \\ St.John College of \\ Engineering \\ Mumbai, India
}

\author{
Angelin Florence \\ Computer Department \\ St.John College of \\ Engineering \\ Mumbai, India
}

\begin{abstract}
The number of vehicles is increasing rapidly in urban areas and metropolitan cities with in crease in population. To manage such a huge amount of traffic in an efficient way is the need of the hour. The conventional traffic signalling system are not adaptable to change in the amount of traffic. This paper solves the problem by making traffic signals smart enough to distinguish between the lanes which have different densities of traffic present in them. This paper makes use of image processing with the help of stored data set of respective lane images and a microprocessor to compute an output.
\end{abstract}

\section{Keywords}

Raspberry $\mathrm{Pi}$, Canny Edge Detection, OpenCV, Image Processing, Python.

\section{INTRODUCTION}

This paper focuses on the solution to the problem generated due to increase in the traffic volume in urban areas. This paper makes traffic signalling smart and efficient for urban areas and metropolitan cities. The complete project is divided into five modules (i.e. Image acquisition from data-set, Image preprocessing, Image processing, Decision making, Output). The data-set image will be sourced from the storage and converted to greyscale image, later this image will be processed by the microprocessor (Raspberry Pi) with the help of OpenCV, a library of Python for Image Processing. The project will make use of binary images captured at real-time and a reference image which will be stored in the system. Thereafter, capturing the image the comparison will be made between the two binary images (i.e. captured image and reference image). The similar comparison will be performed on each lane and then the suitable output will be obtained from the process. If two or more lanes have same amount of traffic then the conventional time interval method will be used to clear the traffic on those lanes.

Different techniques had been proposed in the past such as infrared sensor, induction loop etc. to control traffic but these techniques had their drawbacks. In recent years, image processing has shown excellent outcomes in acquiring real time traffic information using camera module installed along the traffic light.

\section{LITERATURE SURVEY}

Prof. Vikram Deshmukh, Shruti Pantawane, Sonali Hajare, Anushree Kale proposed a system in which the conventional traffic light system has a fixed time pattern which doesn't vary as per the current traffic situation at the junction. This project helped sorting the problem by capturing and converting the lanes images into greyscale images. Threshold value is calculated which will indicate the number of vehicles present at the particular lane. The project uses Image Processing using MATLAB software and microcontroller AT89S52.

The advantages are the use of microcontroller preventing the
The next important advantage is use of MATLAB which is mobile and efficient. The drawbacks that were encountered were the cost of implementation regarding the hardware, use of interpreted language MATLAB which is slow and the chances of microcontroller getting crashed due to excessive load.

D. Prakash, B. Sandhya Devi, R. Naveen Kumar, S. Thiyagarajan, P. Shabarinath proposed a system in which the number of vehicles was counted by the microcontroller. The microcontroller takes decision based on different vehicles count and updates the traffic light delays as a result. The processor used is the ARM architecture. The main advantage of the system is the information like number of vehicles on each lane will be known because of the sensors. The drawbacks that can be marked were the expensive sensors which incur lot of maintenance and the system is scalable.

K. Vidhya, A. Bazila Banu proposed a system, in which the image captured in the traffic signal is processed and converted into greyscale followed by the calculation of threshold value indicating the number of vehicles present on that particular road. After this calculation, it came with the result to understand in which side the density is high. Raspberry Pi is used as a microcontroller for controlling the signals. The main advantages that are evident is firstly the use of OpenCV, secondly the usage of fast and low RAM usage. Also, the hardware level is feasible because use of Raspberry Pi. The disadvantages that can be figured out are that the OpenCV is not that flexible as compared to MATLAB. Also, the Raspberry Pi has a limited amount of memory.

\section{EXISTING SYSTEM}

In the existing system, the conventional traffic system the green light time is assigned to every lane of the road which is pre-defined defined and fixed. It doesn't vary as per the amount of traffic present on the road at that time. Priority factor is missed and the signals are meant for just controlling the assumed amount of traffic rather than computing the realtime traffic scenario.

It is not an efficient way to handle the varying amount of traffic present on the road in metropolitan, smart cities. Equal green light time or predefined amount of time can lead to more congestion on the busy lanes whereas the lanes with the less amount of traffic are given excess green light time which goes in vain. This also leads to frustration among the drivers and the rush leads to accidents sometimes.

This existing system does not even consider the fact that the number of vehicles is directly proportional to congestion of roads where the priority factor is missing and not considered. 


\section{PROPOSED SYSTEM}

The proposed system aims to make the traffic signal more efficient and feasible. Therefore, reducing traffic as well as utilizing the time.

The proposed system will be density based that means it will give priority to the lane which has comparatively a greater number of vehicles. Image processing is the method that will be used for calculating the density. Raspberry Pi will be used as a processor. Images will be acquired using the data set. Acquired images will go through the steps of image processing. Now, the differences will be compared and priorities will be assigned.

The project can be divided into the following modules:

\subsection{Image Acquisition from Data-Set}

An image is taken from the data set of images of road having various amount of vehicle density. Images from the data-set demonstrate the real-time images of traffic.

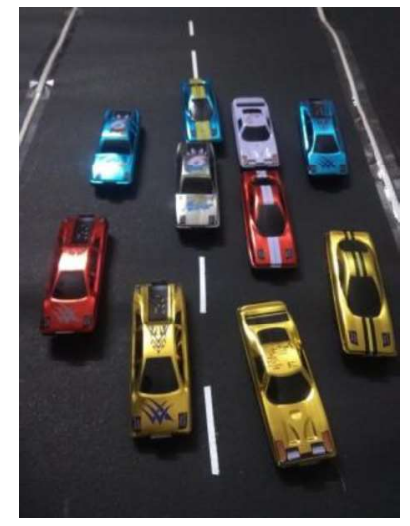

Figure 1: Input acquired from data set

\subsection{Image Pre-Processing}

Pre-processing is basically used for removing unwanted noise and objects from image. The following are the steps that are to be taken in this phase:

Step 1: Greyscale Conversion is the first step in which the RGB image is converted into greyscale image for improving performance. The grey colour image consists of pixel intensities between $0-255$ where 0 represents black pixel and 255 represents white pixel.

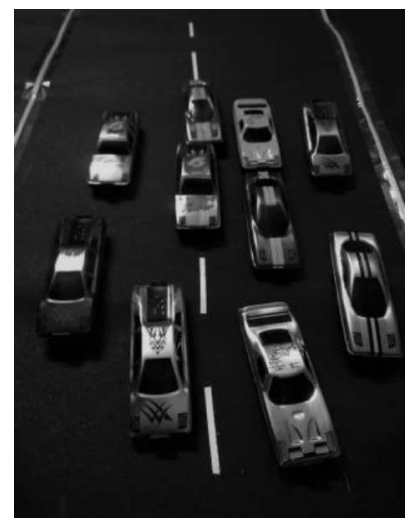

Figure 2: Greyscale Image
Step 2: Thresholding converts the greyscale image into the binary image. If pixel value is greater than a threshold value it is assigned one value (white), else it is assigned the other one. (black).

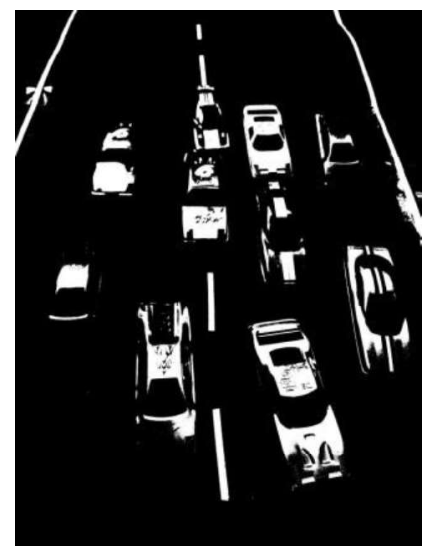

Figure 3: Image after pre-processing

\subsection{Image Processing}

The main purpose of this phase is to serve the processing part on the pre-processed image. The following steps are taken:

Edge detection focuses on the discontinuities in an image. All the edges within the image are detected using Canny Edge Detection algorithm. It provides:
a. Noise Reduction
b. Finding Intensity Gradient

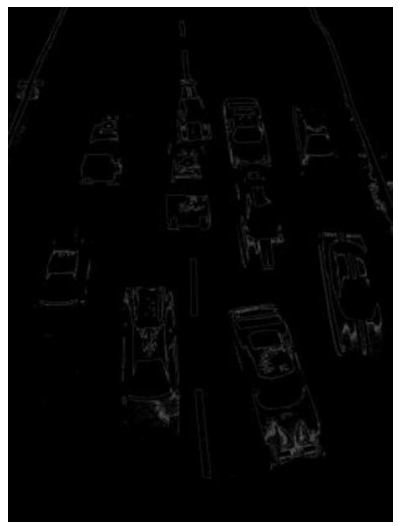

Figure 4: Edge detected image

\subsection{Counting the Non-Zero pixels:}

This step includes counting the number of non-zero pixels i.e. the white pixel count of an acquired images of four lanes from the data-set and the reference image of respective image.

\subsection{Decision Making:}

Here, the value of difference is sorted and priorities are assigned to each lane as per the difference count obtained in the previous step.

The table below tabulates an example of such priority distribution : 


\begin{tabular}{|c|c|c|c|}
\hline Lane & $\begin{array}{c}\text { Differen } \\
\text { ce }\end{array}$ & Priority & $\begin{array}{l}\text { Green Signal } \\
\text { Period }\end{array}$ \\
\hline $\begin{array}{c}\text { Lane } \\
1\end{array}$ & 103405 & 1 & 60 seconds \\
\hline $\begin{array}{c}\text { Lane } \\
2\end{array}$ & 32391 & 4 & 15 seconds \\
\hline $\begin{array}{c}\text { Lane } \\
3\end{array}$ & 47975 & 3 & 30 seconds \\
\hline $\begin{array}{c}\text { Lane } \\
4\end{array}$ & 67484 & 2 & 45 seconds \\
\hline
\end{tabular}

\section{SYSTEM ARCHITECTURE}

\subsection{Raspberry $P i$}

The system uses raspberry $\mathrm{Pi} 3 \mathrm{~B}+$ as it is small, portable and powerful enough to perform all the necessary tasks that are Image Processing, decision making, etc. Raspberry pi $3 \mathrm{~B}+$ has a $1.4 \mathrm{GHz}$ 64-bit quad-core processor, dual-band wireless LAN, Bluetooth 4.2/BLE in it.

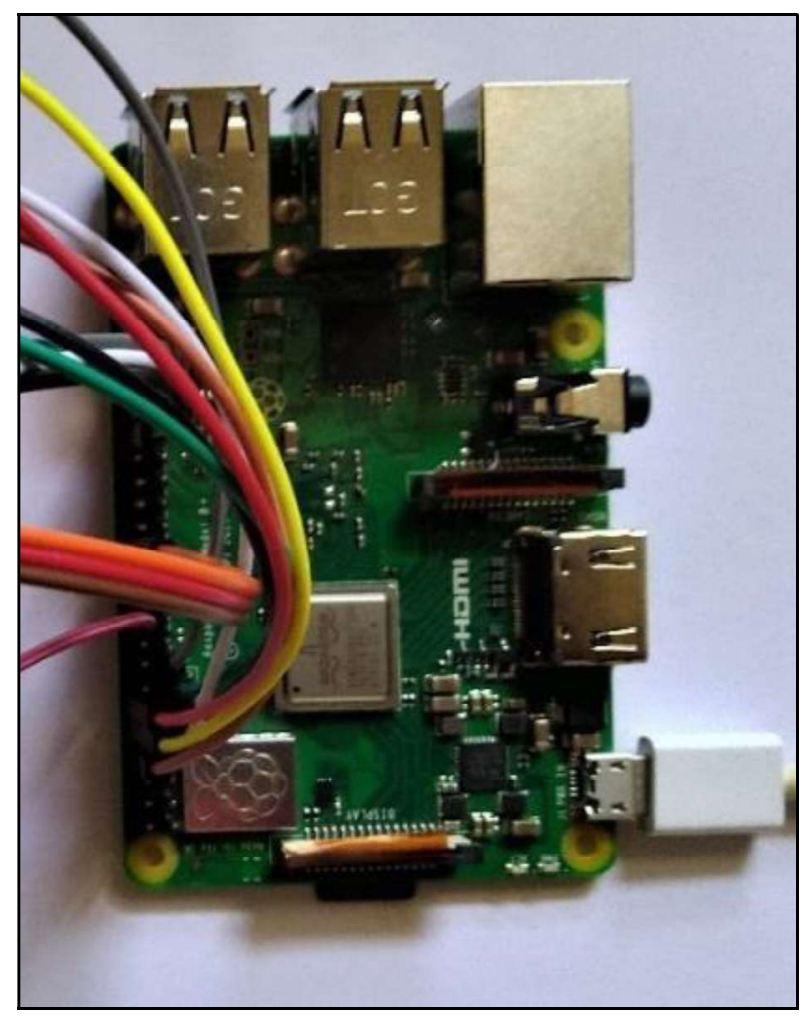

Figure8: Raspberry PßB+

\subsection{Traffic Lights}

LED lights are being used in this system for traffic signal because it consumes less power, have increased light output, last significantly longer, and in the event of an individual LED failure, still operate albeit with a reduced light output. Figure shows the four set of three LEDs which represent four lanes.

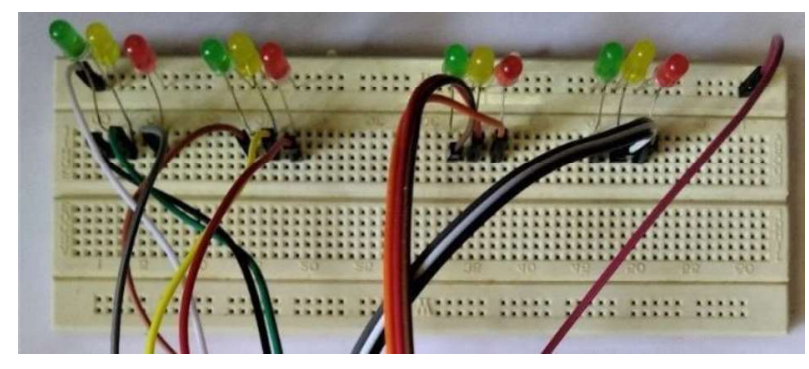

Figure 9: LEDs representing four lanes

\subsection{Power Supply}

Raspberry Pi uses a special 5V DC supply.

\section{RESULT}

When the first set of four images of four lanes at a junction point are acquired from the data set (Viz shown in Figure 6.1), it can be seen that Lane Number 1 has the highest priority as it has highest number of vehicles present on it. The second priority is given to Lane number 4 and so on priority 3 to Lane Number 3 and priority 4 to Lane Number 2 .

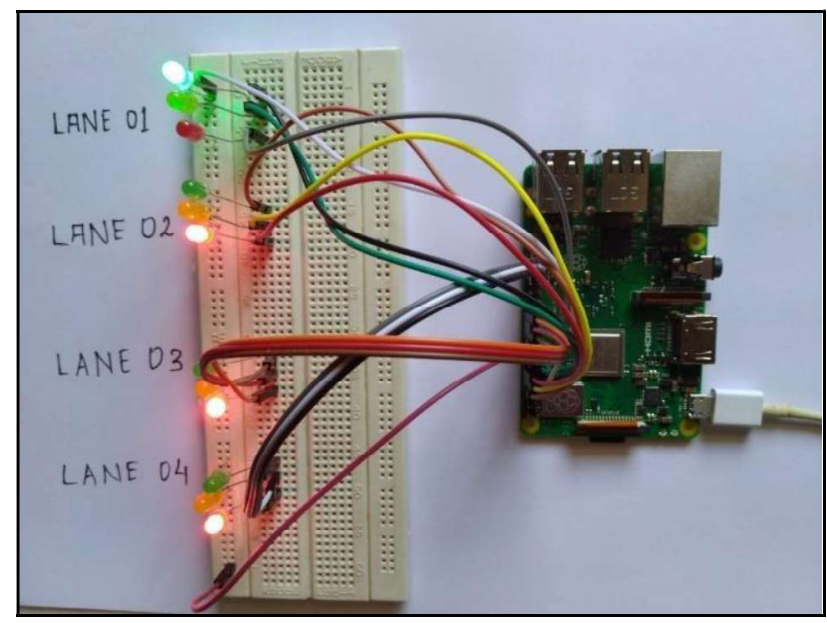

Figure 10: Lane Number 1 Green For 45sec. (Priority 1)

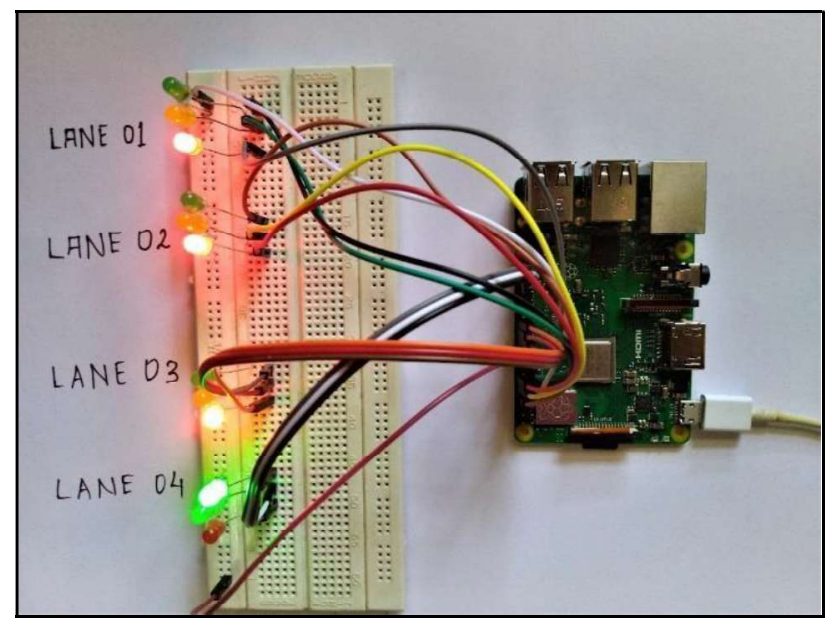

Figure 11: Lane Number 4 Green for 25sec. (Priority 2) 


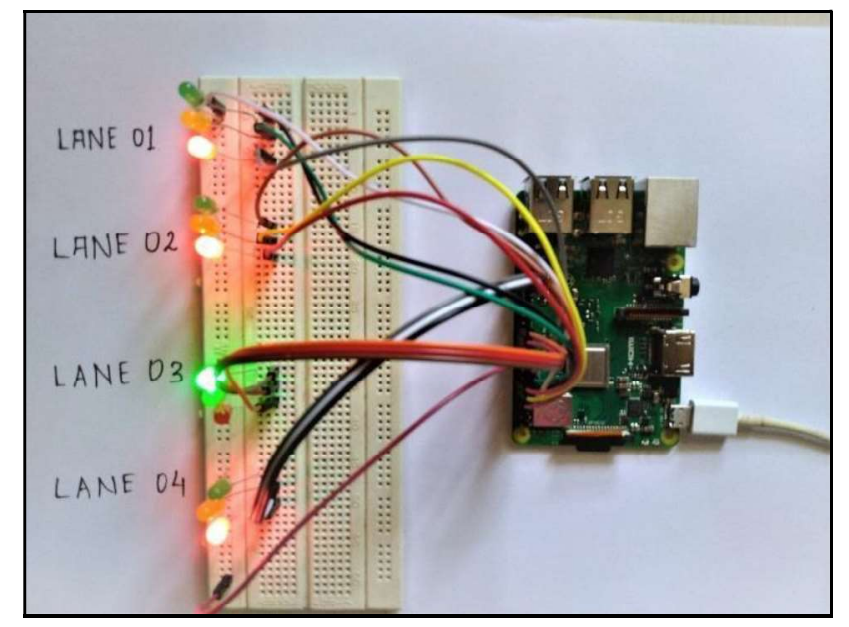

Figure 12: Lane Number 3 Green for 15sec. (Priority 3)

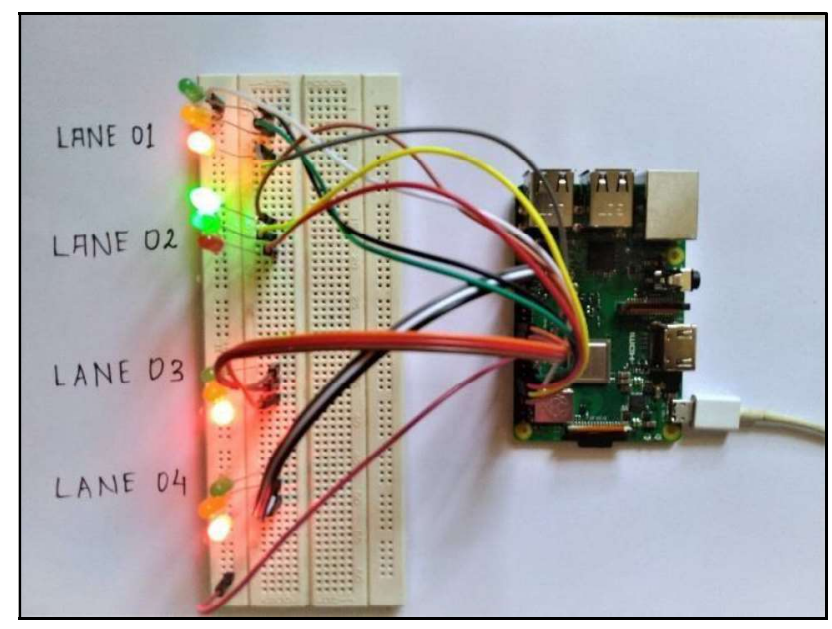

Figure 13: Lane Number 2 Green for 10sec. (Priority 4)

Later after all the four lanes at the junction are given a green light time, all the signals are turned red for pedestrians to cross the road.

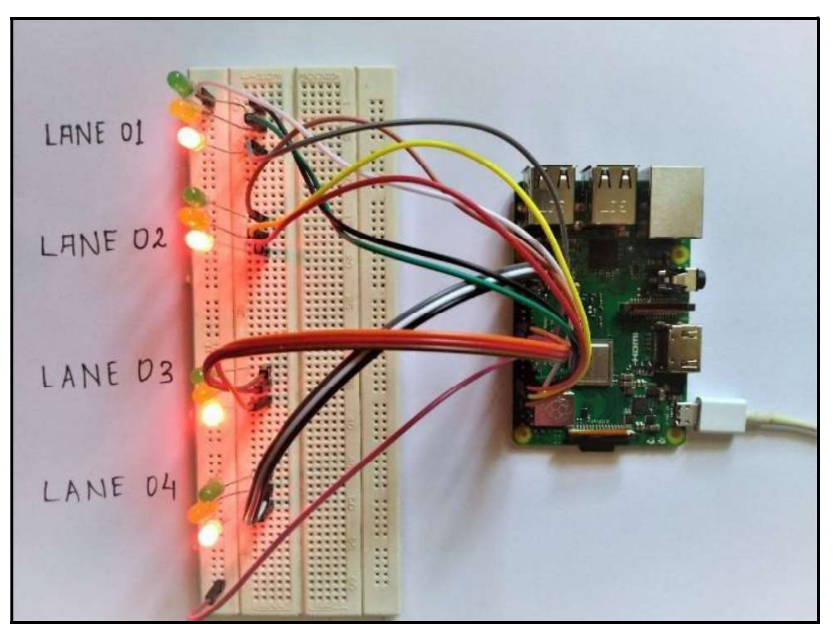

Figure 14: All signals are made red for pedestrians.
Later another set of images are acquired from the data set and the next iteration is continued.

\section{DISCUSSION}

The existing systems makes use use of high cost sensors and/or hardware components that require high maintenance and high budget. whereas the proposed system is cost efficient and stand alone as it does not rely on any special hardware and requires less maintenance as compared to existing system.

\section{CONCLUSION}

Density Based Traffic Signalling System provides a more efficient replacement to the current conventional traffic system. The system uses Image processing to compute the densities and accordingly provide the green light signal to the lanes. The system is an advantage to the denser traffic lanes providing ease of movement, avoiding congestion, also the pedestrians can safely cross the roads and the system is available 24/7. The system is a standalone design and meant to maintain its computation as long as it is connected to the power supply. Also, the design of the system considers the cost factor so as to provide efficient system in way less cost. Using this method at each crossway could help in a continuous journey of the people. This project focuses on making the standard traffic signal density based. This will make the traffic signal more feasible by giving green light time to the lane which has more density.

\section{FUTURE WORK}

Future work can include the feature where we can monitor the signals and the status can be updated at the server. This will help in future reference for generating patterns in the traffic congestion in terms of specific days, holidays, time, etc. It can also be used to prioritize emergency vehicles distinguished by a siren at the top. This may provide an extra priority factor to ambulances, fire brigades, etc.

\section{REFERENCES}

[1] Ashwini D. Bharade, Surabhi S. Gaopande, Robust and Adaptive Traffic Surveillance System for Urban Intersections on Embedded Platform, 2014 Annual IEEE India Conference (INDICON)

[2] Cyrel O.Manlises, Jesus M. Martinez Jr. , Jackson L. Belenzo,Czarleine K. Perez,Maria Khristina Theresa A. Postrero, Real-Time Integrated CCTV Using Face and Pedestrian Detection Image Processing Algorithm For Automatic Traffic Light Transitions, 8thIEEE International Conference Humanoid, Nanotechnology, Information Technology Communication and Control, Environment and Management (HNICEM) The Institute of Electrical and Electronics Engineers Inc. (IEEE) - Philippine Section

[3] Heba A. Kurdi, Review of Closed Circuit Television (CCTV) Techniques for Vehicles Traffic 